\title{
ON WEIGHTED $\beta$-ABSOLUTE CONVERGENCE OF DOUBLE FOURIER SERIES
}

\author{
K. N. DARJI AND R. G. VYAS
}

Abstract. In this paper, we obtain a sufficient condition for the weighted $\beta$-absolute convergence $(0<\beta<2)$ of the double Fourier series of a function $f$ of $(\phi, \psi)-\left(\Lambda^{1}, \Lambda^{2}\right)$-bounded variation.

Mathematics subject classification (2020): Primary 42B05, 42A20; Secondary 26B30, 26D15.

Keywords and phrases: Absolute convergence, double Fourier series, functions of $(\phi, \psi)-\left(\Lambda^{1}, \Lambda^{2}\right)$ bounded variation.

\section{REFERENCES}

[1] A. N. BAKHVALOv, Fourier coefficients of functions from many-dimensional classes of bounded $\Lambda$ variation, Moscow Univ. Math. Bulletin, 66 (1) (2011), 8-16.

[2] K. N. DARJI AND R. G. VyAS, On weighted absolute convergence of multiple Fourier series of a function of $\left(p_{1}, \ldots, p_{N}\right)-\left(\Lambda^{1}, \ldots, \Lambda^{N}\right)-B V$, Acta Math. Hungar., 156 (2) (2018), 361-371.

[3] L. Gogoladze And R. Meskhia, On the absolute convergence of trigonometric Fourier series, Proc. Razmadze. Math. Inst., 141 (2006), 29-40.

[4] F. MóRICZ AND A. Veres, Absolute convergence of multiple Fourier series revisited, Anal. Math., 34 (2) (2008), 145-162.

[5] F. Móricz AND A. Veres, On the absolute convergence of multiple Fourier series, Acta Math. Hungar., 117 (3) (2007), 275-292.

[6] M. Schramm AND D. Waterman, Absolute convergence of Fourier series of functions of class $\Lambda B V^{(p)}$ and $\varphi \Lambda B V$, Acta. Math. Acad. Sci. Hungar., 40 (3-4) (1982), 273-276.

[7] P. L. UL'YANOV, Series with respect to a Haar system with monotone coefficients (in Russian), Izv. Akad. Nauk. SSSR Ser. Mat., 28 (1964), 925-950.

[8] R. G. VYAS, On the absolute convergence of Fourier series of functions of $\Lambda B V^{(p)}$ and $\varphi \Lambda B V$, Georgian Math. J., 14 (4) (2007), 769-774.

[9] R. G. VyAS AND K. N. DARJi, On absolute convergence of multiple Fourier series, Math. Notes, 94 (1) (2013), 71-81; Russian transl., Mat. Zametki, 94 (1) (2013), 81-93.

[10] R. G. VyAS AND K. N. DARJI, On multiple Fourier coefficients of functions of $\phi-\Lambda$-bounded variation, Math. Inequal. Appl., 17 (3) (2014), 1153-1160.

[11] R. G. Vyas AND K. N. DARJi, Order of magnitude of multiple Fourier coefficients, Anal. Theory Appl., 29 (1) (2013), 27-36.

[12] R. G. Vyas And J. R. Patadia, On the absolute convergence of Fourier series of functions of generalized bounded variations, J. Indian Math. Soc., 62, (1-4) (1996), 129-136. 